\title{
Solar Activity Monitoring of Flares and CMEs Precursors through Lyman-Alpha Imaging and Tracking of Filaments and Prominences
}

\author{
Luc Damé ${ }^{1}$ and Safinaz A. Khaled ${ }^{2,1}$ \\ ${ }^{1}$ LATMOS/IPSL/CNRS/UVSQ, 11 Boulevard d'Alembert, 78280 Guyancourt, France \\ email: luc.dame@latmos.ipsl.fr \\ ${ }^{2}$ Space Weather Monitoring Center, Helwan University, Helwan, Egypt \\ email: safinaz_1986@yahoo.com
}

\begin{abstract}
We investigate the advantages of imaging solar filaments and prominences in LymanAlpha, coupled to H-Alpha on ground, to develop more reliable precursors indicators for large flares, several hours before their occurrence.
\end{abstract}

Keywords. Solar flares, filaments, ultraviolet.

\section{Rationale}

Events preceding the onset of a flare are called 'precursors', and one of the prominent precursors is a newly emerging bipolar region at the surface, which may interact with pre-existing magnetic field in the corona and trigger a flare. Another well-known precursor is the activation, or eruption, of a filament that is composed of relatively cool plasma (around $10000 \mathrm{~K}$ ), floating in the hot coronal plasma. Both emerging regions and filaments are very well observed in Lyman-Alpha (in Space) and H-Alpha (on ground and formed lower in the solar atmosphere), both on the disk and at the limb, and we expect that their combination can lead to better identification of changes at the origin of major eruptions and most important coronal mass ejections (CMEs).

Lyman-Alpha can provide early (precursor) detection on the disk, hours before the event, of filament/prominence eruptions (better than the He II line, well suited only for limb observations). As studied by Mierla et al. (2013), the imaging of an erupting prominence shows that the acceleration at the top of the prominence increases smoothly and continuously with height (slow rise), and this over hours $(4-5)$ before the eruption. This indeed indicates that prominences are not accelerated immediately by local reconnection but are rather swept away (modifying shape, helicity) as part of a large-scale relaxation of the coronal magnetic field.

\section{Observations and results}

As shown in Fig. 1, the Lyman-Alpha signal (observed with LYRA/PROBA-2) peaks during the rising phase of the flare and has a very significant level. This M2.0 flare of February 8, 2010, was analyzed in detail by Kretzschmar et al. (2013). It indeed shows that Lyman-Alpha is very sensitive to flares, 1000 times more than H-Alpha formed lower in the atmosphere; the signature on the light curve of this M2 flare is reaching almost $1 \%$ in integrated light of the solar disk! The Lyman-Alpha line, the most intense solar line, is obviously very sensitive to flares and temperature variations in the chromosphere (see Milligan et al. 2011) but also to velocities and magnetic fields (Zeeman effect). It 


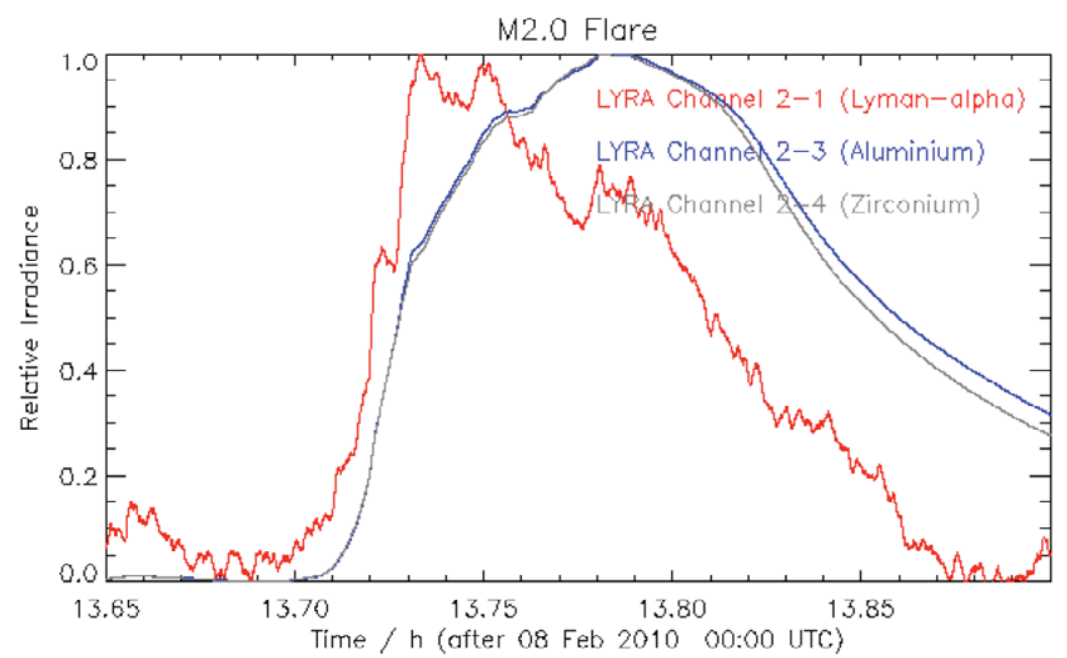

Figure 1. During a flare, the Lyman-Alpha signal, cooler, peaks during the rising phase, slightly earlier than X-rays or XUV (early LYRA/PROBA-2 data with proper Lyman-Alpha channel). has the advantage to be much thicker than the H-Alpha line (cf. Labrosse et al. 2010) and to be formed higher in the solar atmosphere. By combining imaging (and velocities if possible) in both Lyman and H-Alpha, one can expect to better assess the filament 3D shape and its evolution up to the disruption/eruption "disparition brusque".

Lyman-Alpha has both the capacity of the early detection of a large flare/CME by imaging the filament change/acceleration, and the detection sensitivity of the flare/CME occurrence, even better than soft X-rays (GOES measurements) or XUV and EUV bands (as illustrated on Fig. 1).

Lyman-Alpha imaging, in that respect, would be a very high value Space Weather product complementing EUV imaging available on several satellites.

\section{Conclusion}

These considerations on Lyman-Alpha and H-Alpha observations are pointing the interest for future Space Lyman-Alpha irradiance measurements coupled, for precursors identification, to solar disk imaging, as proposed by the SWUSV (Space Weather \& Ultraviolet Solar Variability) Microsatellite Mission (Damé and The SWUSV Team 2013).

\section{Acknowledgements}

We are grateful to I. Dammasch and M. Dominique for access to and use of LYRA/PROBA2 data, to S. UeNo for the H-Alpha data of Hida Observatory, and to R. Kariyappa and S.T. Kumara for help with the data analysis (image segmentation).

\section{References}

Damé, L., The SWUSV Team 2013, Journal of Adv. Research, 4, 235

Kretzschmar, M., Dominique, M., \& Dammasch, I. E. 2013, Solar Phys., 286, 221

Labrosse, N., Heinzel, P., Vial, J.-C., Kucera, T., Parenti, S., Gunár, S., Schmieder, B., \& Kilper, G. 2010, Space Sci. Rev., 151, 243

Mierla, M., Heinzel, P., Seaton, D. B., Berghmans, D., Chifu, I., De Groof, A., Inhester, B., Rodriguez, L., Stenborg, G., \& Zhukov, A. N. 2013, Solar Phys., 286, 241

Milligan, R. O., Chamberlin, P., Hudson, H., Woods, T., Mathioudakis, M., Fletcher, L., Kowalski, A., \& Keenan, F. 2011, ApJ., 748, L14 